\title{
$\beta$-Arrestin 1 in Thyrotropin Receptor Signaling in Bone: Studies in Osteoblast-Like Cells
}

OPEN ACCESS

Edited by:

Francesco S. Celi,

Virginia Commonwealth University,

United States

Reviewed by:

Lei Zhang,

Cardiff University, United Kingdom

Akira Sugawara,

Tohoku University, Japan

*Correspondence:

Susanne Neumann

susannen@intra.niddk.nih.gov

Specialty section:

This article was submitted to

Thyroid Endocrinology,

a section of the journal

Frontiers in Endocrinology

Received: 03 February 2020

Accepted: 24 April 2020

Published: 20 May 2020

Citation:

Boutin A, Gershengorn MC and Neumann S (2020) $\beta$-Arrestin 1 in Thyrotropin Receptor Signaling in Bone: Studies in Osteoblast-Like Cells. Front. Endocrinol. 11:312.

doi: $10.3389 /$ fendo.2020.00312

\author{
Alisa Boutin, Marvin C. Gershengorn and Susanne Neumann* \\ Laboratory of Endocrinology and Receptor Biology, National Institute of Diabetes and Digestive and Kidney Diseases, \\ National Institutes of Health, Bethesda, MD, United States
}

A direct action of thyrotropin (TSH, thyroid-stimulating hormone) on bone precursors in humans is controversial. Studies in rodent models have provided conflicting findings. We used cells derived from a moderately differentiated osteosarcoma stably overexpressing human TSH receptors (TSHRs) as a model of osteoblast precursors (U2OS-TSHR cells) to investigate TSHR-mediated effects in bone differentiation in human cells. We review our findings that (1) TSHR couples to several different G proteins to induce upregulation of genes associated with osteoblast activity-interleukin 11 (IL-11), osteopontin (OPN), and alkaline phosphatase (ALPL) and that the kinetics of the induction and the $G$ protein-mediated signaling pathways involved were different for these genes; (2) TSH can stimulate $\beta$-arrestin-mediated signal transduction and that $\beta$-arrestin 1 in part mediates TSH-induced pre-osteoblast differentiation; and (3) TSHR/insulin-like growth factor 1 (IGF1) receptor (IGF1R) synergistically increased OPN secretion by TSH and IGF1 and that this crosstalk was mediated by physical association of these receptors in a signaling complex that uses $\beta$-arrestin 1 as a scaffold. These findings were complemented using a novel $\beta$-arrestin 1-biased agonist of TSHR. We conclude that TSHR can signal via several transduction pathways leading to differentiation of this model system of human pre-osteoblast cells and, therefore, that TSH can directly regulate these bone cells.

Keywords: $\beta$-arrestin 1, TSH receptor, IGF1 receptor, osteoblast-like cells, positive allosteric modulator, crosstalk

\section{INTRODUCTION}

Thyroid-stimulating hormone (TSH), also known as thyrotropin, is a hormone that activates TSH receptors (TSHRs) to stimulate development of the thyroid in utero and on thyroid cells to stimulate production of thyroid hormones thyroxine (T4) and triiodothyronine (T3) in the adult. Thyroid hormones are essential for skeletal development and healthy bone metabolism $(1,2)$. Clinical studies have demonstrated that euthyroid status in the adult is important for bone homeostasis. Hyperthyroidism leads to increased bone resorption which causes reduced bone and its mineralization. Graves' hyperthyroidism presents an increased risk for osteoporosis $(3,4)$. Patients with hypothyroidism exhibit reduced bone turnover as osteoclast activity is reduced. The effects of hyperthyroidism, hypothyroidism, and subclinical hyperthyroidism on bone metabolism have been extensively reviewed $(2,5,6)$.

While the pivotal role of thyroid hormones in bone homeostasis has been well documented, the role of TSH itself is still under study. The question if there is a direct, thyroid hormone-independent action of TSH on bone is of specific interest. TSHR expression has been demonstrated in rodent 
osteoblasts and osteoclasts (2). TSHR knockout causes osteoporosis and focal osteosclerosis in mice (7). TSH administration inhibits bone loss in adult, ovariectomized rodents consistent with the idea that TSH is an activator of bone formation (8). Overall, in vivo studies in mice and rats propose $\mathrm{TSH}$ as a fine-tuning regulator of bone homeostasis, and these findings have been comprehensively reviewed $(1,2,6,7,9,10)$.

Several clinical studies have also pointed to direct action of TSH on bone. Administration of recombinant human TSH (rhTSH) in postmenopausal women increased serum Nterminal propeptide of type-I procollagen (PINP), a marker of bone formation, demonstrating an anabolic effect of TSH in humans (11). Mazziotti et al. showed that short-term rhTSH stimulation leads to a reversible inhibition of bone resorption in postmenopausal women suggesting a role for TSH in patients with bone loss and a high bone turnover rate (12). Furthermore, epidemiological studies demonstrated a tight relationship between low TSH levels and parameters of bone loss and fracture risk [reviewed in (4)]. However, the role of TSH on the adult skeleton and its mechanisms of action in human bone have yet to be defined in more detail and are still controversial [reviewed in $(1,2,4,6)$ ].

TSHR is expressed in human bone, however, TSH is not expressed in primary human osteoblasts or osteoclasts (13). Pituitary TSH works systemically, and therefore, it is likely that it can activate TSHRs in bone. Thyrostimulin, an ancestral glycoprotein hormone and TSHR agonist, has been considered as a regulator of bone formation. In contrast to TSH, thyrostimulin is expressed in osteoblasts and osteoclasts (14). The combination of in vivo and in vitro studies demonstrated a role for thyrostimulin during skeletal development but an unessential role in the adult skeleton (14).

In human thyrocytes, TSHR coupling to $\mathrm{G} \alpha_{\mathrm{s}}$ and activation of the cAMP-protein kinase A (PKA) signal transduction system has been considered the primary pathway of TSH regulation (15). In vitro studies in bone cells were initially hampered by the assumption that TSH-induced cAMP production is the primary TSHR-mediated signaling pathway. TSH stimulation of some bone cells did not result in cAMP production, and therefore, at first, a potential physiological role of TSH in bone was underestimated. However, recent studies have shown that other $G$ protein- and $\beta$-arrestin-mediated signaling pathways can be activated via TSHR and the quest for the role of TSH in bone metabolism gained traction again. TSHR activates mitogen-activated protein kinase 1/3 (ERK1/2) (16), p38 mitogen-activated protein kinase 1 (p38 MAPK) (17), and AKT serine/threonine kinase 1 (AKT1) (18). The activation of these three kinases by other $G$ protein-coupled receptors (GPCRs) is known to require, in part, $\beta$-arrestin involvement (19). Parathyroid hormone (PTH), a key regulator of bone metabolism acting through the parathyroid hormone 1 receptor (PTH1R), translocates both $\beta$-arrestin 1 and 2 and activates ERK1/2 (20). Our group has demonstrated that TSH mediates activatory TSHR signaling through $\beta$-arrestin 1 , and that this pathway plays an important role in stimulating upregulation of osteoblast markers driving the precursors toward an osteoblast phenotype (21).
This review will focus on TSH signaling in human osteoblast precursor cells and summarize the roles of $G$ protein- and $\beta$ arrestin-mediated signaling pathways with a special emphasis on the role of $\beta$-arrestin 1 in bone physiology.

\section{G PROTEIN-MEDIATED TSHR SIGNALING IN U2OS-TSHR CELLS}

The majority of studies on the TSH effect on bone homeostasis were performed in mice or mouse cell cultures $(7,8,22-24)$. We used U2OS cells derived from a moderately differentiated osteosarcoma stably overexpressing human TSHR (U2OS-TSHR cells) as a model of osteoblast precursors (21) to investigate TSHR-mediated effects in bone differentiation in human cells. U2OS cells had previously been shown to exhibit differentiation toward osteoblasts (25). Parental U2OS cells express very low endogenous TSHR; TSHR mRNA in U2OS cells is four orders of magnitude lower than in U2OS-TSHR cells. Treatment of parental U2OS cells with TSH did not stimulate cAMP production or osteoblast marker upregulation (21).

TSHR is known to couple to multiple G proteins (26). This capability to couple to and activate multiple $G$ protein-mediated signaling pathways is also exhibited by the PTH1R (27), which is highly expressed on osteoblasts. PTH1R can signal via several signaling pathways including cAMP to activate PKA and $\mathrm{G} \alpha_{\mathrm{q} / 11}$ to activate phospholipase C.

We demonstrated that TSHR couples to several different $\mathrm{G}$ proteins to induce upregulation of genes associated with osteoblast activity in U2OS-TSHR cells: interleukin 11 (IL-11) $(28,29)$, osteopontin (OPN) (30), and alkaline phosphatase (ALPL) (31). The kinetics of the induction and the signaling pathways involved, however, were different for these genes (32).

IL-11 upregulation was rapid and reached its maximum within $4 \mathrm{~h}$ of treatment with TSH. IL-11 expression was primarily regulated by the $\mathrm{G} \alpha_{\mathrm{s}}$-cAMP pathway. The conclusion that TSH regulated IL-11 expression via the $\mathrm{G} \alpha_{\mathrm{s}}$-cAMP pathway was based on the observation that forskolin (FSK), an adenylyl cyclase activator, upregulated IL-11, and H-89, a PKA inhibitor, inhibited TSH-mediated IL-11 upregulation. Silencing of $G \alpha_{\mathrm{S}}$ by siRNA inhibited TSH-mediated upregulation of IL-11 as well as basal IL-11 expression, most likely due to inhibition of TSHR's constitutive $\mathrm{G} \alpha_{\mathrm{s}}$-mediated signaling activity.

By contrast, we found that OPN induction by TSH was independent of the $\mathrm{G} \alpha_{\mathrm{s}}$ pathway as neither FSK nor H-89 had a significant effect. OPN induction was slower than that of IL-11 requiring $72 \mathrm{~h}$ for detection. Pertussis toxin (PTX), which inhibits $\mathrm{G} \alpha_{\mathrm{o}}$ i protein activation, inhibited OPN stimulation by TSH. The knockdown of $\mathrm{G} \alpha_{\mathrm{i} 1,2,3}$ confirmed that $\mathrm{G}_{\mathrm{i}}$ is important for TSHinduced OPN upregulation. In addition, we showed that p38 MAPK phosphorylation was necessary for OPN upregulation. This likely occurred downstream of $\mathrm{G}_{\mathrm{i}}$ because TSH-mediated phosphorylation of p38 MAPK was inhibited by PTX, and siRNA knockdown of p38 MAPK and LY2228820, a p38 MAPK kinase inhibitor, caused suppression of OPN also.

We determined that ALPL induction required approximately $24 \mathrm{~h}$ for detection and exhibited a biphasic dose response curve 
with lower doses of TSH resulting in decreased levels of ALPL and higher doses stimulating its upregulation. Interestingly, the $\mathrm{EC}_{50}$ for cAMP stimulation was similar to that of downregulation of ALPL. Furthermore, activation of the cAMP pathway by FSK downregulated basal levels of ALPL whereas inhibition of this signaling cascade by $\mathrm{H}-89$ and $\mathrm{G} \alpha_{\mathrm{s}}$ knockdown increased basal ALPL levels. Conversely, the $\mathrm{EC}_{50}$ for $\mathrm{G} \alpha_{\mathrm{q} / 11}$ pathway activation was similar to that of ALPL upregulation. We had shown previously that higher doses of TSH were required for $\mathrm{G} \alpha_{\mathrm{q} / 11}$ activation as two TSH molecules were necessary to bind to the TSHR homodimer in order to activate $\mathrm{G} \alpha_{\mathrm{q} / 11}$ signaling (33). The knockdown of $\mathrm{G} \alpha_{\mathrm{q} / 11}$ confirmed that $\mathrm{G} \alpha_{\mathrm{q} / 11}$ signaling is involved in ALPL upregulation by TSH. We showed that ALPL upregulation was mediated in part by protein kinase $\mathrm{C}$ (PKC), which is activated downstream of $\mathrm{G} \alpha_{\mathrm{q} / 11}$ by diacylglycerol, because the PKC inhibitor GF109203X inhibited ALPL upregulation, and the PKC activator phorbol 12,13dibutyrate upregulated ALPL. Lastly, since the dose responses for ERK1/2 phosphorylation and ALPL upregulation were similar, we used U0126, a mitogen-activated protein kinase kinase 7 (MEK) inhibitor, to show that ERK1/2 inactivation also inhibited ALPL induction. Combined, our findings indicate that regulation of ALPL by TSH is biphasic. The higher potency (lower doses) TSH effect to downregulate ALPL is mediated by the G $\alpha_{\mathrm{s}}$-cAMP pathway whereas the lower potency (higher doses) TSH effect to upregulate ALPL is mediated in part by the $\mathrm{G} \alpha_{\mathrm{q} / 11}$-PKC-ERK1/2 signaling cascade.

Thus, only upregulation of IL-11, a cytokine required for normal bone remodeling, was mainly mediated via cAMP, the traditionally recognized "primary" signaling pathway for TSH. By contrast, ALPL was inhibited by cAMP at low $\mathrm{TSH}$ concentrations. It appears that unlike in thyrocytes, in which cAMP is a positive modulator of thyroid cell differentiation $(34,35)$, in U2OS-TSHR cells, cAMP is a negative modulator of differentiation and is only one of several pathways involved in osteoblast differentiation. These findings help us to better understand the multipathway nature of TSHR signaling revealing that it is more complex and cell context-dependent than previously understood (32).

Of note, thyrostimulin induced responses similar to those of TSH in regulation of IL11, OPN and ALPL (32). Although it appears that TSH is acting as a hormone to reach the site(s) of action in the bone via the bloodstream, thyrostimulin may play a role in anabolic regulation in bone in a paracrine manner during early skeletal development, as has been demonstrated in mice (14).

\section{$\beta$-ARRESTIN-MEDIATED SIGNALING IN U2OS-TSHR CELLS}

It has been shown that the anabolic effects of PTH are mediated via a $\beta$-arrestin signal transduction pathway independent of $G$ protein signaling. In the case of PTH1R, the primary activator of the anabolic effect is $\beta$-arrestin 2 , which was demonstrated using a $\beta$-arrestin-biased agonist (36). In contrast, nonselective

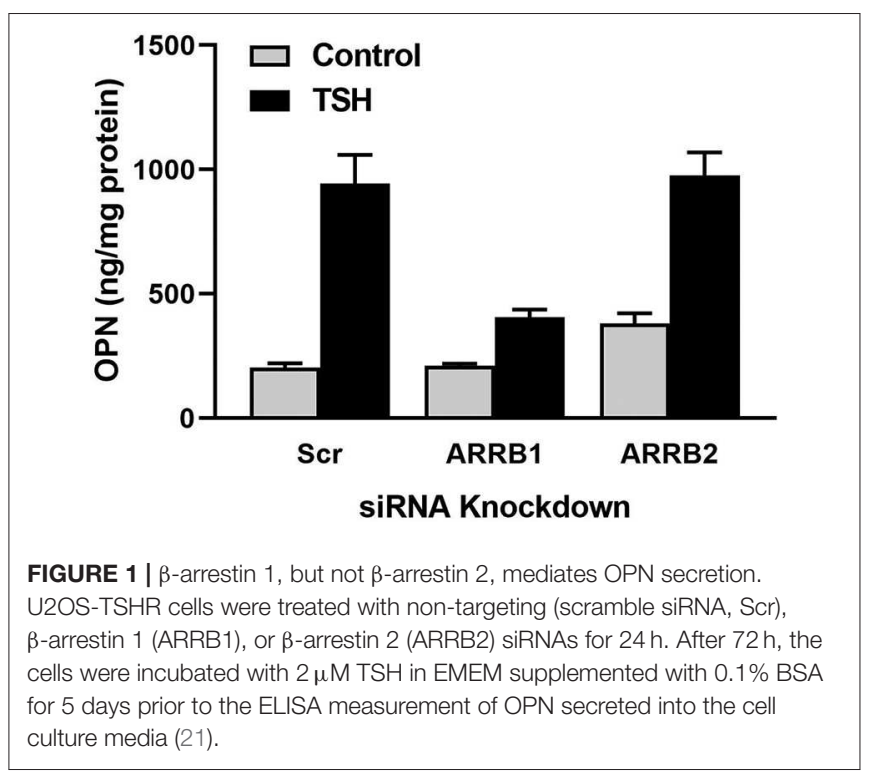

$\mathrm{PTH}$ also mediates osteoblast-osteoclast coupling via $\mathrm{G} \alpha_{\mathrm{s}} / \mathrm{cAMP}$ promoting bone turnover $(37,38)$.

We investigated whether TSH can stimulate $\beta$-arrestin mediated signal transduction in U2OS-TSHR cells (21). In addition to $\mathrm{TSH}$, we utilized a small molecule ligand $\mathrm{C} 2$ (NCGC00161870) that is functionally selective for cAMP pathway activation (39). We demonstrated that TSHR stimulation with TSH, but not $\mathrm{C} 2$, leads to phosphorylation of ERK1/2, AKT1, and p38 MAPK phosphokinases. Similarly, we found that $\mathrm{TSH}$, but not $\mathrm{C} 2$, stimulated upregulation of osteoblast markers such as ALPL, OPN, and tumor necrosis factor superfamily member 11 (RANKL). Using the DiscoveRx PathHunter $\beta$-arrestin protein complementation assay, we found that TSH induced translocation of both $\beta$-arrestin 1 and 2 to the TSHR while C2, despite being fully efficacious for cAMP production, did not. Even though TSH stimulated translocation of both $\beta$-arrestins, siRNA-mediated silencing of $\beta$-arrestins demonstrated that only $\beta$-arrestin 1 was necessary for phosphorylation of ERK1/2, AKT1, and p38 MAPK phosphokinases. In contrast, we observed an increase in phospho-AKT1 and ERK1/2 with $\beta$-arrestin 2 knockdown. Frenzel et al. (40) showed that $\beta$-arrestin 2 was predominantly involved in TSHR desensitization, which may explain why $\beta$-arrestin 2 knockdown caused an increase in AKT1 and ERK1/2 phosphorylation. Lastly, we examined involvement of $\beta$-arrestins in mediation of TSH-induced expression of osteoblastic markers. Knockdown of $\beta$-arrestin 1, but not $\beta$-arrestin 2 , inhibited upregulation of RANKL and OPN. Figure 1 illustrates that the $\beta$-arrestin 1 pathway accounts for $\sim 80 \%$ of TSH-stimulated OPN upregulation. The remaining $20 \%$ is most likely mediated by $\mathrm{G}$ proteins, in particular $\mathrm{G} \alpha_{\mathrm{i}}$ (32). $\beta$-arrestin 2 does not play a role in OPN secretion (Figure 1). Thus, in U2OS-TSHR cells, $\beta$-arrestin 1 mediates TSHR-induced activatory signals, whereas $\beta$-arrestin 2 appears to be a mediator of inhibitory signals and desensitization. The fact that $\beta$-arrestin 1 in part 

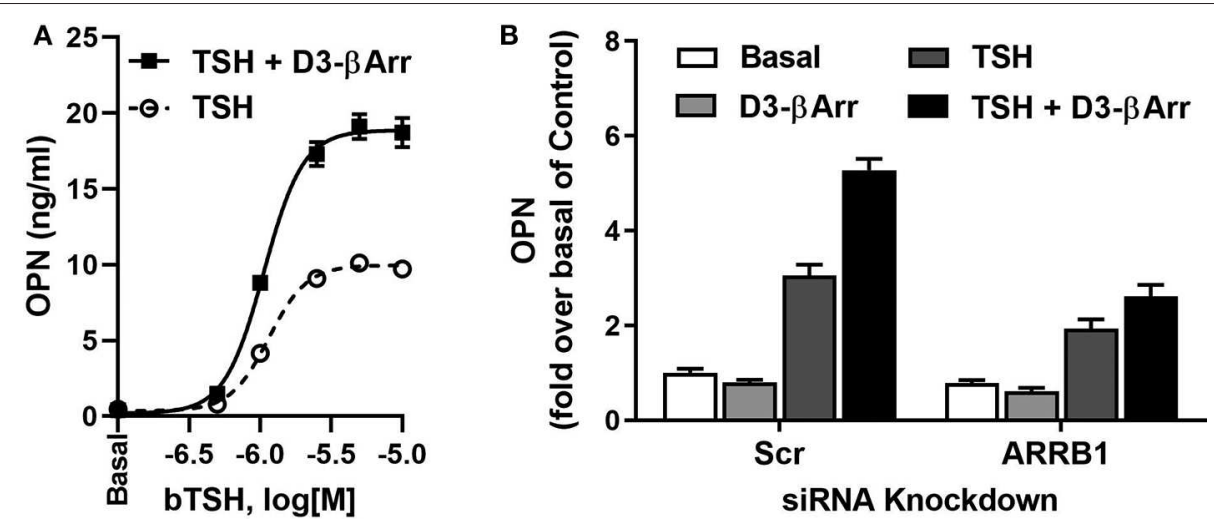

FIGURE 2 | D3- $\beta$ Arr is a functionally selective TSHR agonist. (A) D3- $\beta$ Arr is a positive allosteric modulator for TSH-induced OPN secretion. U2OS-TSHR cells were treated with increasing doses of TSH with or without $5 \mu \mathrm{M}$ D3- $\beta$ Arr in EMEM with $0.1 \%$ BSA for 7 days prior to OPN measurement by ELISA in cell culture media (43). (B) The potentiating effect of D3- $\beta$ Arr is mediated by $\beta$-arrestin 1 in U2OS-TSHR cells. Cells were transfected with non-targeting (Scr) or $\beta$-arrestin 1 (ARRB1) siRNA, respectively. After $72 \mathrm{~h}$ of siRNA-mediated knockdown, cells were treated with $10 \mu \mathrm{M}$ D3- $\beta$ Arr, $2 \mu \mathrm{M}$ TSH or their combination in EMEM with $0.1 \%$ BSA for 5 days. OPN secretion was measured in conditioned media by ELISA (43).
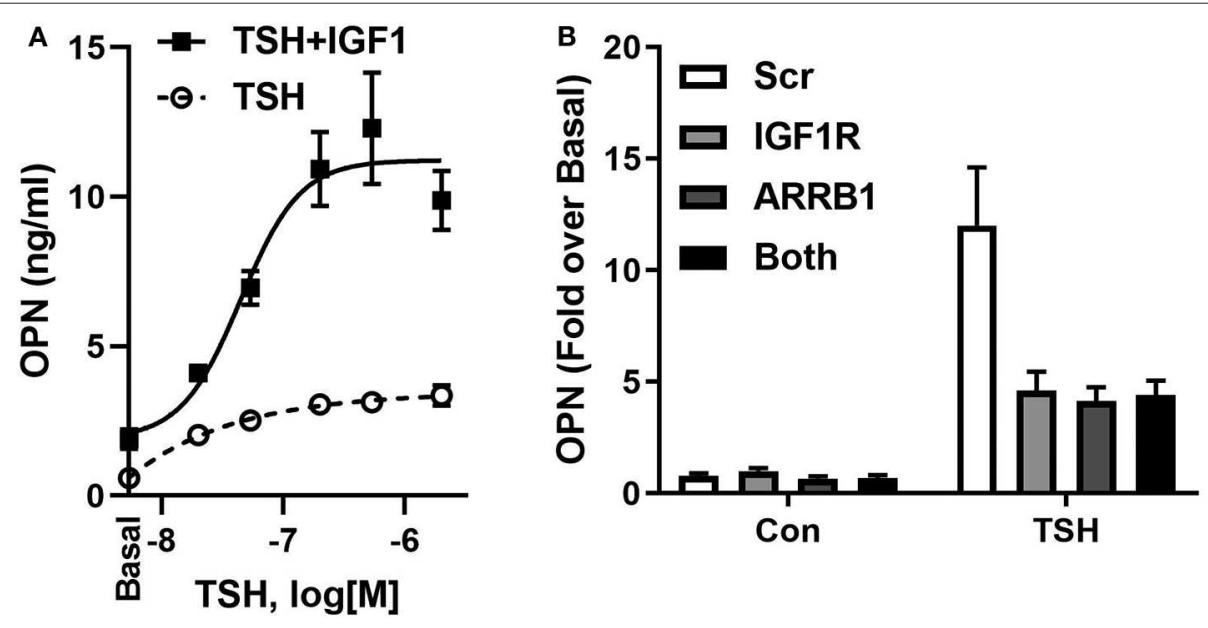

FIGURE 3 | TSHR and IGF1R exhibit crosstalk in U2OS-TSHR cells. (A) TSH and IGF1 synergistically stimulate OPN secretion. U2OS-TSHR cells were treated with increasing doses of recombinant human TSH (rhTSH) with or without $100 \mathrm{ng} / \mathrm{ml}$ IGF1. After 5 days, OPN secreted into cell culture media was measured by ELISA. This original figure is based on the data published in (52). (B) IGF1R and $\beta$-arrestin 1 are part of the same signaling pathway in TSHR/IGF1R crosstalk-mediated OPN secretion. U2OS-TSHR cells, in which IGF1R and $\beta$-arrestin1 (ARRB1) were silenced separately or simultaneously were treated with 10 mU/ml rhTSH. After 5 days, OPN was measured in culture media and compared to scramble siRNA (Scr) control. Knockdown efficiencies of IGF1R and ARRB1 were 95.5 and 82.9\%, respectively (52).

mediates TSH-induced osteoblast differentiation indicates that this pathway may play an anabolic role in bone physiology (21).

The potencies for TSH-induced translocation of both $\beta$ arrestins to the TSHR were approximately 16-fold lower than that for CAMP stimulation and similar to that for stimulation of phosphoinositide signaling $(33,41)$. As mentioned above, we previously demonstrated that these differences in potency could be attributed to the fact that for $G \alpha_{s}$ coupling leading to adenylyl cyclase activation and cAMP production binding of a single molecule of TSH to the TSHR homodimer (or oligomer) was sufficient. However, coupling to $\mathrm{G} \alpha_{\mathrm{q} / 11}$ and subsequent activation of phospholipase $\mathrm{C}$ and phosphoinositide signaling required binding of two TSH molecules to a TSHR homodimer (33). Even though evidence has been presented that a number of GPCRs form heterodimers that can interact with $\beta$-arrestins for internalization, it remains controversial whether homodimerization is required for binding to $\beta$-arrestins (42). Nonetheless, to explain the lower potency of TSH to stimulate translocation of $\beta$-arrestins to TSHR compared to cAMP stimulation, we suggest that translocation is enhanced by binding of two TSH molecules to a TSHR homodimer (21).

To study TSH-enhanced osteoblast differentiation more deeply, we sought to discover a drug-like agonist that is selective for $\beta$-arrestin 1 pathway activation by TSHR. D3$\beta$ Arr (NCGC00379308) was identified through high throughput screening and selected out of 368,816 compounds because 
A

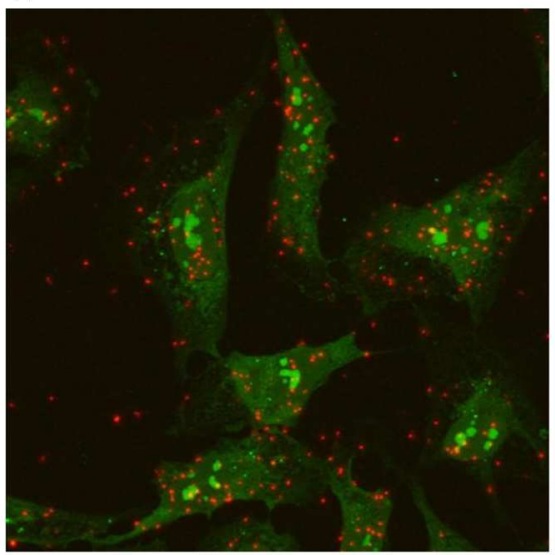

B

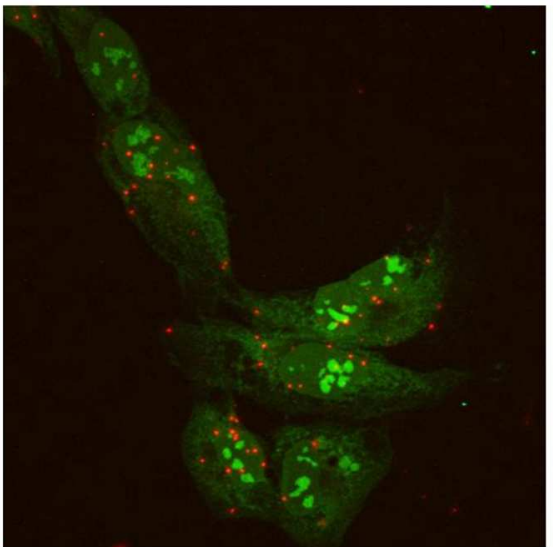

C

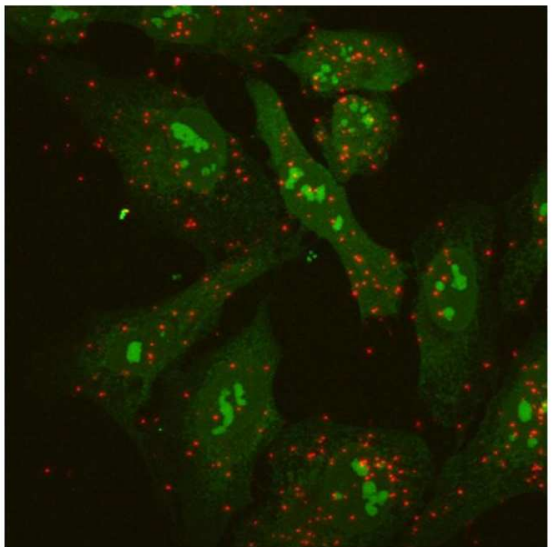

FIGURE 4 | TSHR/IGF1R crosstalk is dependent on receptor proximity and $\beta$-arrestin 1 stabilizes the protein complex. TSHR/IGF1R signaling complexes were detected using a Proximity Ligation Assay (PLA) and visualized with fluorescent microscopy. A PLA signal is measured when TSHR and IGF1R are in a protein complex within $40 \mathrm{~nm}$ of each other. PLA was performed in unstimulated U2OS-TSHR cells treated with non-targeting (A), $\beta$-arrestin 1 (ARRB1) (B), or $\beta$-arrestin 2 (ARRB2) (C) siRNA. Positive PLA signals are shown in red. SYTO9 nucleic acid staining, applied to determine the cell number, is shown in green at $\times 63$ magnification. The knock-down of $\beta$-arrestin 1 reduced the number of positive PLA signals by $55 \%$ demonstrating this proteins stabilizing function in the TSHR/IGF1R complex (52).

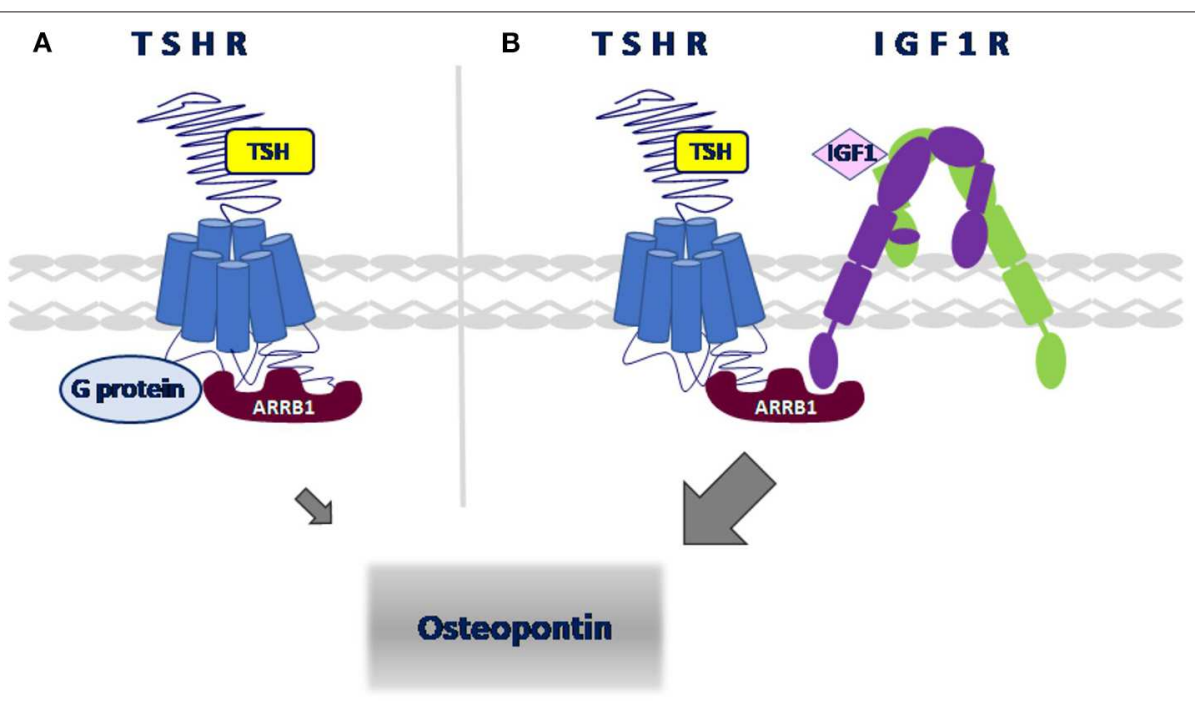

FIGURE 5 | Model of signaling pathways leading to OPN secretion in U2OS-TSHR cells. (A) TSHR-mediated upregulation of OPN. TSH-induced OPN secretion is regulated via $\mathrm{G} \alpha_{i}$ and $\beta$-arrestin 1-mediated signal transduction pathways. (B) $\beta$-arrestin 1 scaffolds TSHR and IGF1R to enable crosstalk. TSH and IGF1 synergistically upregulate OPN secretion. $\beta$-arrestin 1 stabilizes the receptor complex which enables TSHR/IGF1R crosstalk.

it caused translocation of $\beta$-arrestin 1 to the TSHR without stimulation of cAMP production (43). Thus, D3- $\beta$ Arr is a functionally selective TSHR ligand. D3- $\beta$ Arr stimulated $\beta$-arrestin 1 translocation more effectively than TSH and potentiated the effect of TSH in stimulating $\beta$-arrestin 1 translocation. D3- $\beta$ Arr also stimulated $\beta$-arrestin 2 translocation but with decreased potency and efficacy compared to $\beta$-arrestin 1 . The functional selectivity of D3- $\beta$ Arr allowed us to suggest that this drug-like ligand may be used as a probe to enhance $\beta$-arrestin 1 -mediated signaling over $G$ protein-dependent signaling in follow-up studies on its role in bone physiology.
Furthermore, we studied whether the effect of D3- $\beta$ Arr on $\beta$-arrestin 1 translocation and/or potentiation of TSH would translate into enhancement of TSH-induced upregulation of osteoblast genes. D3- $\beta$ Arr by itself did not increase OPN mRNA but upregulated ALPL mRNA 4-fold. Most importantly, D3- $\beta$ Arr enhanced TSH stimulation of OPN secretion (Figure 2A). siRNA knockdowns of $\beta$-arrestin 1 and 2 confirmed that $\beta$-arrestin 1 mediated the potentiating effect of D3- $\beta$ Arr on TSH-stimulated OPN secretion (Figure 2B). Thus, we established that D3- $\beta$ Arr is a positive allosteric modulator of TSH-induced upregulation of osteoblast genes and OPN secretion in a human in vitro 
cell model. D3- $\beta$ Arr or an analog may serve to probe TSHR physiology in bone in vivo in the future (43).

\section{TSHR/IGF1R CROSSTALK AND THE ROLE $\beta$-ARRESTIN 1 AS A SCAFFOLD OF THIS PROTEIN COMPLEX IN U2OS-TSHR CELLS}

Crosstalk (or transactivation) between GPCRs and receptor tyrosine kinases is an established signaling mechanism $(44,45)$. Crosstalk between TSHR and insulin-like growth factor 1 (IGF1) receptor (IGF1R) has recently been studied in the context of the pathogenesis of Graves' ophthalmopathy (46-49) as well as thyroid specific gene upregulation in human primary thyrocytes (50). We examined whether this phenomenon occurred in U2OSTSHR cells.

IGF1 and IGF1R were shown to play important roles in bone development and in regulation of bone homeostasis in the adult (51). In U2OS-TSHR cells, we observed that IGF1 alone mildly stimulated OPN but, more importantly, TSH and IGF1 synergized to upregulate OPN secretion (Figure 3A). Individual knockdowns of $\beta$-arrestin 1 and IGF1R resulted in inhibition of OPN secretion to similar extents. Their co-knockdown did not show any additive effect (Figure 3B), consistent with the idea that these proteins are in the same signaling pathway. Moreover, the IGF1R inhibitor linsitinib decreased TSH-mediated OPN secretion even in the absence of IGF1 (52). These data support the idea of TSHR/IGF1R crosstalk in U2OS-TSHR cells and suggested a potential role for $\beta$-arrestin 1 in this protein complex (52).

We used the Proximity Ligation Assay (PLA) to show that TSHR and IGF1R were held within $40 \mathrm{nM}$ or less of each other by $\beta$-arrestin 1 . Knockdown of $\beta$-arrestin 1 in U2OSTSHR cells exhibited a $55 \%$ decrease $\left({ }^{*} P<0.0001\right)$ in PLApositive signals per cell while knockdown of $\beta$-arrestin 2 had no effect (Figure 4). These results provide evidence that $\beta$-arrestin 1 is associated with TSHR and IGF1R under unstimulated

\section{REFERENCES}

1. Baliram R, Latif R, Zaidi M, Davies TF. Expanding the role of thyroidstimulating hormone in skeletal physiology. Front Endocrinol. (2017) 8:252. doi: 10.3389/fendo.2017.00252

2. Bassett JH, Williams GR. Role of thyroid hormones in skeletal development and bone maintenance. Endocr Rev. (2016) 37:13587. doi: 10.1210/er.2015-1106

3. Kisakol G, Kaya A, Gonen S, Tunc R. Bone and calcium metabolism in subclinical autoimmune hyperthyroidism and hypothyroidism. Endocr J. (2003) 50:657-61. doi: 10.1507/endocrj.50.657

4. Zaidi M, Lizneva D, Kim SM, Sun L, Iqbal J, New MI, et al. FSH, bone mass, body fat, and biological aging. Endocrinology. (2018) 159:350314. doi: 10.1210/en.2018-00601

5. Tuchendler D, Bolanowski M. The influence of thyroid dysfunction on bone metabolism. Thyroid Res. (2014) 7:12. doi: 10.1186/s13044-0140012-0

6. Williams GR, Bassett JHD. Thyroid diseases and bone health. J Endocrinol Invest. (2018) 41:99-109. doi: 10.1007/s40618-017-0753-4 conditions. Moreover, since $\beta$-arrestin 1 knockdown inhibited the synergistic stimulation of OPN secretion, these data demonstrate that close proximity of TSHR and IGF1R is required for receptor crosstalk, and that this physical proximity in a protein complex depends on the presence of $\beta$-arrestin 1 . Figure 5 illustrates a model of TSHR-mediated OPN secretion mediated by $\mathrm{G}$ proteins and $\beta$-arrestin 1 as well as the role of $\beta$-arrestin 1 as a central scaffold in allowing for TSHR/IGF1R crosstalk leading to further increase of OPN secretion and U2OSTSHR cell differentiation (52).

\section{CONCLUSION}

In this review, we summarized our findings showing that the role of $\beta$-arrestins in TSHR signal transduction in osteoblast-like cells is more prominent than previously understood. In addition to canonical receptor desensitization and internalization, $\beta$-arrestin 1, along with $\mathrm{G}$ proteins, mediates TSH-enhanced osteoblast differentiation. Specifically, $\beta$-arrestin 1 serves as a physical scaffold to enable TSHR/IGF1R crosstalk (Figure 5). We also describe how the $\beta$-arrestin 1-biased agonist D3- $\beta$ Arr, a small molecule positive allosteric modulator for TSHR, may be used to better understand the role of TSHR in bone differentiation. Since TSHR may signal via $\beta$-arrestin 1 in humans in a clinically relevant manner, our data suggest that a TSHR positive allosteric modulator may have a potential role as a therapy for osteoporosis.

\section{AUTHOR CONTRIBUTIONS}

All authors have contributed significantly to the work, have read the manuscript, attest to the validity and legitimacy of the data and its interpretation, and agree to its submission.

\section{FUNDING}

This work was funded by the National Institute of Diabetes and Digestive and Kidney Diseases Intramural Research Program Z01 DK047045.
7. Abe E, Marians RC, Yu W, Wu XB, Ando T, Li Y, et al. TSH is a negative regulator of skeletal remodeling. Cell. (2003) 115:15162. doi: 10.1016/S0092-8674(03)00771-2

8. Sun L, Vukicevic S, Baliram R, Yang G, Sendak R, McPherson $\mathrm{J}$, et al. Intermittent recombinant TSH injections prevent ovariectomy-induced bone loss. Proc Natl Acad Sci USA. (2008) 105:4289-94. doi: 10.1073/pnas.0712395105

9. Abe E, Sun L, Mechanick J, Iqbal J, Yamoah K, Baliram R, et al. Bone loss in thyroid disease: role of low TSH and high thyroid hormone. Ann N Y Acad Sci. (2007) 1116:383-91. doi: 10.1196/annals.1402.062

10. de Lloyd A, Bursell J, Gregory JW, Rees DA, Ludgate M. TSH receptor activation and body composition. J Endocrinol. (2010) 204:13-20. doi: 10.1677/JOE-09-0262

11. Martini G, Gennari L, De Paola V, Pilli T, Salvadori S, Merlotti D, et al. The effects of recombinant TSH on bone turnover markers and serum osteoprotegerin and RANKL levels. Thyroid. (2008) 18:45560. doi: 10.1089/thy.2007.0166

12. Mazziotti G, Sorvillo F, Piscopo M, Cioffi M, Pilla P, Biondi B, et al. Recombinant human TSH modulates in vivo C-telopeptides of type-1 collagen 
and bone alkaline phosphatase, but not osteoprotegerin production in postmenopausal women monitored for differentiated thyroid carcinoma. $J$ Bone Miner Res. (2005) 20:480-6. doi: 10.1359/JBMR.041126

13. Bassett JH, Williams AJ, Murphy E, Boyde A, Howell PG, Swinhoe R, et al. A lack of thyroid hormones rather than excess thyrotropin causes abnormal skeletal development in hypothyroidism. Mol Endocrinol. (2008) 22:50112. doi: 10.1210/me.2007-0221

14. Bassett JH, van der Spek A, Logan JG, Gogakos A, Bagchi-Chakraborty J, Williams AJ, et al. Thyrostimulin regulates osteoblastic bone formation during early skeletal development. Endocrinology. (2015) 156:3098-113. doi: 10.1210/en.2014-1943

15. Latif R, Morshed SA, Zaidi M, Davies TF. The thyroid-stimulating hormone receptor: impact of thyroid-stimulating hormone and thyroidstimulating hormone receptor antibodies on multimerization, cleavage, and signaling. Endocrinol Metab Clin North Am. (2009) 38:319-41, viii. doi: 10.1016/j.ecl.2009.01.006

16. Iacovelli L, Capobianco L, Salvatore L, Sallese M, D’Ancona GM, De Blasi A. Thyrotropin activates mitogen-activated protein kinase pathway in FRTL5 by a cAMP-dependent protein kinase A-independent mechanism. Mol Pharmacol. (2001) 60:924-33. doi: 10.1124/mol.60.5.924

17. Pomerance M, Abdullah HB, Kamerji S, Correze C, Blondeau JP. Thyroidstimulating hormone and cyclic AMP activate $\mathrm{p} 38$ mitogen-activated protein kinase cascade. Involvement of protein kinase $\mathrm{A}, \mathrm{racl}$, and reactive oxygen species. J Biol Chem. (2000) 275:40539-46. doi: 10.1074/jbc.M002097200

18. Turcu AF, Kumar S, Neumann S, Coenen M, Iyer S, Chiriboga P, et al. A small molecule antagonist inhibits thyrotropin receptor antibody-induced orbital fibroblast functions involved in the pathogenesis of Graves ophthalmopathy. J Clin Endocrinol Metab. (2013) 98:2153-9. doi: 10.1210/jc.2013-1149

19. DeWire SM, Ahn S, Lefkowitz RJ, Shenoy SK. Betaarrestins and cell signaling. Annu Rev Physiol. (2007) 69:483510. doi: 10.1146/annurev.physiol.69.022405.154749

20. Gesty-Palmer D, Chen M, Reiter E, Ahn S, Nelson CD, Wang S, et al. Distinct beta-arrestin- and $\mathrm{G}$ protein-dependent pathways for parathyroid hormone receptor-stimulated ERK1/2 activation. J Biol Chem. (2006) 281:1085664. doi: 10.1074/jbc.M513380200

21. Boutin A, Eliseeva E, Gershengorn MC, Neumann S. beta-Arrestin-1 mediates thyrotropin-enhanced osteoblast differentiation. FASEB J. (2014) 28:344655. doi: 10.1096/fj.14-251124

22. Sampath TK, Simic P, Sendak R, Draca N, Bowe AE, O'Brien S, et al. Thyroid-stimulating hormone restores bone volume, microarchitecture, and strength in aged ovariectomized rats. J Bone Miner Res. (2007) 22:84959. doi: $10.1359 /$ jbmr.070302

23. Baliram R, Latif R, Berkowitz J, Frid S, Colaianni G, Sun L, et al. Thyroidstimulating hormone induces a Wnt-dependent, feed-forward loop for osteoblastogenesis in embryonic stem cell cultures. Proc Natl Acad Sci USA. (2011) 108:16277-82. doi: 10.1073/pnas.1110286108

24. Baliram R, Sun L, Cao J, Li J, Latif R, Huber AK, et al. Hyperthyroid-associated osteoporosis is exacerbated by the loss of TSH signaling. J Clin Invest. (2012) 122:3737-41. doi: 10.1172/JCI63948

25. Salvatori L, Caporuscio F, Coroniti G, Starace G, Frati L, Russo MA, et al. Down-regulation of epidermal growth factor receptor induced by estrogens and phytoestrogens promotes the differentiation of U2OS human osteosarcoma cells. J Cell Physiol. (2009) 220:35-44. doi: 10.1002/jcp.21724

26. Laugwitz KL, Allgeier A, Offermanns S, Spicher K, Van Sande J, Dumont JE, et al. The human thyrotropin receptor: a heptahelical receptor capable of stimulating members of all four G protein families. Proc Natl Acad Sci USA. (1996) 93:116-20. doi: 10.1073/pnas.93.1.116

27. Bohinc BN, Gesty-Palmer D. Arrestins in bone. Prog Mol Biol Transl Sci. (2013) 118:335-58. doi: 10.1016/B978-0-12-394440-5.00013-9

28. Leng SX, Elias JA. Interleukin-11. Int J Biochem Cell Biol. (1997) 29:105962. doi: 10.1016/S1357-2725(97)00017-4

29. Sims NA, Jenkins BJ, Nakamura A, Quinn JM, Li R, Gillespie MT, et al. Interleukin-11 receptor signaling is required for normal bone remodeling. $J$ Bone Miner Res. (2005) 20:1093-102. doi: 10.1359/JBMR.050209

30. Chatakun P, Nunez-Toldra R, Diaz Lopez EJ, Gil-Recio C, Martinez-Sarra E, Hernandez-Alfaro F, et al. The effect of five proteins on stem cells used for osteoblast differentiation and proliferation: a current review of the literature. Cell Mol Life Sci. (2014) 71:113-42. doi: 10.1007/s00018-013-1326-0
31. Aubin JE, Liu F, Malaval L, Gupta AK. Osteoblast and chondroblast differentiation. Bone. (1995) $17(2$ Suppl.):77S-83. doi: 10.1016/8756-3282(95)00183-E

32. Boutin A, Neumann S, Gershengorn MC. Multiple transduction pathways mediate thyrotropin receptor signaling in preosteoblast-like cells. Endocrinology. (2016) 157:2173-81. doi: 10.1210/en.2015-2040

33. Allen MD, Neumann S, Gershengorn MC. Occupancy of both sites on the thyrotropin (TSH) receptor dimer is necessary for phosphoinositide signaling. FASEB J. (2011) 25:3687-94. doi: 10.1096/fj.11-188961

34. Roger PP, Christophe D, Dumont JE, Pirson I. The dog thyroid primary culture system: a model of the regulation of function, growth and differentiation expression by cAMP and other well-defined signaling cascades. Eur J Endocrinol. (1997) 137:579-98. doi: 10.1530/eje.0.1370579

35. Tramontano D, Moses AC, Veneziani BM, Ingbar SH. Adenosine 3',' monophosphate mediates both the mitogenic effect of thyrotropin and its ability to amplify the response to insulin-like growth factor I in FRTL5 cells. Endocrinology. (1988) 122:127-32. doi: 10.1210/endo-122-1-127

36. Gesty-Palmer D, Luttrell LM. 'Biasing' the parathyroid hormone receptor: a novel anabolic approach to increasing bone mass? Br J Pharmacol. (2011) 164:59-67. doi: 10.1111/j.1476-5381.2011.01450.x

37. Bisello A, Chorev M, Rosenblatt M, Monticelli L, Mierke DF, Ferrari SL. Selective ligand-induced stabilization of active and desensitized parathyroid hormone type 1 receptor conformations. J Biol Chem. (2002) 277:3852430. doi: $10.1074 /$ jbc.M202544200

38. Ferrari SL, Pierroz DD, Glatt V, Goddard DS, Bianchi EN, Lin FT, et al. Bone response to intermittent parathyroid hormone is altered in mice null for \{beta\}-Arrestin2. Endocrinology. (2005) 146:1854-62. doi: 10.1210/en.2004-1282

39. Neumann S, Huang W, Titus S, Krause G, Kleinau G, Alberobello AT, et al. Small-molecule agonists for the thyrotropin receptor stimulate thyroid function in human thyrocytes and mice. Proc Natl Acad Sci USA. (2009) 106:12471-6. doi: 10.1073/pnas.0904506106

40. Frenzel R, Voigt C, Paschke R. The human thyrotropin receptor is predominantly internalized by beta-arrestin 2. Endocrinology. (2006) 147:3114-22. doi: 10.1210/en.2005-0687

41. Urizar E, Montanelli L, Loy T, Bonomi M, Swillens S, Gales C, et al. Glycoprotein hormone receptors: link between receptor homodimerization and negative cooperativity. EMBO J. (2005) 24:1954-64. doi: 10.1038/sj.emboj.7600686

42. Milligan G. A day in the life of a $\mathrm{G}$ protein-coupled receptor: the contribution to function of $\mathrm{G}$ protein-coupled receptor dimerization. $\mathrm{Br} J$ Pharmacol. (2008) 153 (Suppl. 1):S216-29. doi: 10.1038/sj.bjp.0707490

43. Neumann S, Eliseeva E, Boutin A, Barnaeva E, Ferrer M, Southall N, et al. Discovery of a positive allosteric modulator of the thyrotropin receptor: potentiation of thyrotropin-mediated preosteoblast differentiation in vitro. J Pharmacol Exp Ther. (2018) 364:38-45. doi: 10.1124/jpet.117. 244095

44. Delcourt N, Bockaert J, Marin P. GPCR-jacking: from a new route in RTK signalling to a new concept in GPCR activation. Trends Pharmacol Sci. (2007) 28:602-7. doi: 10.1016/j.tips.2007.09.007

45. Pyne NJ, Pyne S. Receptor tyrosine kinase-G-protein-coupled receptor signalling platforms: out of the shadow? Trends Pharmacol Sci. (2011) 32:44350. doi: 10.1016/j.tips.2011.04.002

46. Krieger CC, Neumann S, Marcus-Samuels B, Gershengorn MC. TSHR/IGF-1R cross-talk, not IGF-1R stimulating antibodies, mediates graves' ophthalmopathy pathogenesis. Thyroid. (2017) 27:746-7. doi: 10.1089/thy.2017.0105

47. Krieger CC, Neumann S, Place RF, Marcus-Samuels B, Gershengorn MC. Bidirectional TSH and IGF-1 receptor cross talk mediates stimulation of hyaluronan secretion by Graves' disease immunoglobins. J Clin Endocrinol Metab. (2015) 100:1071-7. doi: 10.1210/jc.2014-3566

48. Krieger CC, Place RF, Bevilacqua C, Marcus-Samuels B, Abel BS, Skarulis MC, et al. TSH/IGF-1 receptor cross talk in graves' ophthalmopathy pathogenesis. J Clin Endocrinol Metab. (2016) 101:2340-7. doi: 10.1210/jc.2016-1315

49. Tsui S, Naik V, Hoa N, Hwang CJ, Afifiyan NF, Sinha Hikim A, et al. Evidence for an association between thyroid-stimulating hormone and insulin-like growth factor 1 receptors: a tale of two antigens implicated in Graves' disease. J Immunol. (2008) 181:4397-405. doi: 10.4049/jimmunol.181.6.4397 
50. Morgan SJ, Neumann S, Marcus-Samuels B, Gershengorn MC. Thyrotropin and insulin-like growth factor 1 receptor crosstalk upregulates sodium-iodide symporter expression in primary cultures of human thyrocytes. Thyroid. (2016) 26:1794-803. doi: 10.1089/thy.2016.0323

51. Kawai M, de Paula FJ, Rosen CJ. New insights into osteoporosis: the bone-fat connection. J Intern Med. (2012) 272:31729. doi: 10.1111/j.1365-2796.2012.02564.x

52. Krieger CC, Boutin A, Jang D, Morgan SJ, Banga JP, Kahaly GJ, et al. Arrestin-beta-1 physically scaffolds TSH and IGF1 receptors to enable crosstalk. Endocrinology. (2019) 160:1468-79. doi: 10.1210/en.201900055
Conflict of Interest: The authors declare that the research was conducted in the absence of any commercial or financial relationships that could be construed as a potential conflict of interest.

Copyright (C) 2020 Boutin, Gershengorn and Neumann. This is an open-access article distributed under the terms of the Creative Commons Attribution License (CC BY). The use, distribution or reproduction in other forums is permitted, provided the original author(s) and the copyright owner(s) are credited and that the original publication in this journal is cited, in accordance with accepted academic practice. No use, distribution or reproduction is permitted which does not comply with these terms. 